TITLE:

\title{
Contributions of steady heat conduction to the rate of chemical reaction
}

\section{AUTHOR(S):}

Kim, Hyeon-Deuk; Hayakawa, Hisao

\section{CITATION:}

Kim, Hyeon-Deuk ... [et al]. Contributions of steady heat conduction to the rate of chemical reaction. Chemical Physics Letters 2003, 372(3-4): 314-319

ISSUE DATE:

2003-04

URL:

http://hdl.handle.net/2433/49789

\section{RIGHT:}

Copyright (C) 2004 Elsevier B.V. All rights reserved.; この論文は出版社版 でありません。引用の際には出版社版をご確認ご利用ください。; This is not the published version. Please cite only the published version. 


\title{
Contributions of steady heat conduction to the rate of chemical reaction
}

\author{
Kim Hyeon-Deuk*, Hisao Hayakawa \\ Graduate School of Human and Environmental Studies, \\ Kyoto University, Kyoto 606-8501, Japan
}

(Dated: February 26, 2008)

\begin{abstract}
We have derived the effect of steady heat flux on the rate of chemical reaction based on the lineof-centers model using the explicit velocity distribution function of the steady-state Boltzmann equation for hard-sphere molecules to second order. It is found that the second-order velocity distribution function plays an essential role for the calculation of it. We have also compared our result with those from the steady-state Bhatnagar-Gross-Krook(BGK) equation and information theory, and found no qualitative differences among them.
\end{abstract}

PACS numbers: 05.20.Dd, 82.20.-w, 82.20.Pm

\footnotetext{
*kim@yuragi.jinkan.kyoto-u.ac.jp
} 


\section{INTRODUCTION}

Chemical reactions in gases has been studied with the aid of gas kinetic theory [1, 2, 3], 4]. Present proposed the line-of-centers model for chemical reaction in gases, which has been accepted as a standard model to describe the chemical reaction in gases. 4, 15, 6, 7, 8, 9, 10, $11,12,13,14,15,16,17,18,19,20,21]$

Under nonequilibrium situations such as gases under the heat conduction and the shear flow, their nonequilibrium effects on the rate of chemical reaction have attracted attention among researchers. 12, 13, 14, 15, 16, 17, 18, 22, 23] However, to derive the effect of steady heat flux on the rate of chemical reaction in the line-of-centers model, we need the explicit velocity distribution function of the steady-state Boltzmann equation for hard-sphere molecules to second order in density and temperature gradient. To our knowledge, nobody had derived the explicit velocity distribution function of the Boltzmann equation for hardsphere molecules to second order. Although Burnett determined the second-order pressure tensor for the Boltzmann equation [1], he had not derived the explicit second-order velocity distribution function of the Boltzmann equation. 24, 25] This is a result of mathematical difficulties, as was indicated by Fort and Cukrowski. 12, 13] Therefore, none has succeeded to obtain the correct reaction rate of Present's model except for Fort and Cukrowski who adopted information theory [26] as the nonequilibrium velocity distribution function to second order. 12, 13 ]

Recently, we have derived the explicit velocity distribution function of the steady-state Boltzmann equation for hard-sphere molecules to second order. 25] In this letter, we apply the explicit velocity distribution function to the calculation of the rate of chemical reaction in the line-of-centers model under steady heat conduction.

\section{SIMPLE MODEL FOR CHEMICAL REACTION}

In the early stage of a chemical reaction between monatomic molecules:

$$
A+A \rightarrow \text { products }
$$

the rate of chemical reaction is not affected by the existence of products. 27] From the viewpoint of kinetic collision theory 1, 2, 3, 4], the rate of chemical reaction (1) can be 
described as

$$
R=\int d \mathbf{v} \int d \mathbf{v}_{1} \int d \boldsymbol{\Omega} \int f f_{1} g \sigma(g),
$$

where $\mathbf{v}$ and $\mathbf{v}_{1}$ are the velocities of the molecules, $g=\left|\mathbf{v}-\mathbf{v}_{1}\right|$ their relative speed, $\Omega$ the solid angle, $f=f(\mathbf{r}, \mathbf{v})$ and $f_{1}=f\left(\mathbf{r}, \mathbf{v}_{1}\right)$ are the distributions of $\mathbf{v}$ and $\mathbf{v}_{1}$ at $\mathbf{r}$, respectively.

We adopt the line-of-centers model as the differential cross-section of chemical reaction

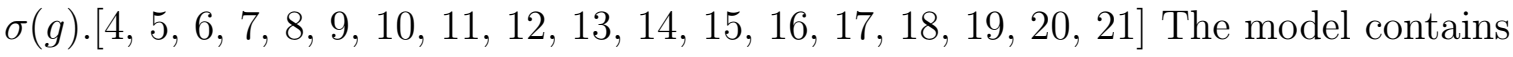

$$
\sigma(g)=\left\{\begin{array}{ll}
0 & g<\sqrt{\frac{4 E^{*}}{m}} \\
\frac{d^{2}}{4}\left(1-\frac{4 E^{*}}{m g^{2}}\right) & g \geq \sqrt{\frac{4 E^{*}}{m}}
\end{array},\right.
$$

with $m$ mass of the molecules and $E^{*}$ the threshold energy of the chemical reaction. $d$ is regarded as a distance between centers of monatomic molecules at contact. [4, 21]

In order to calculate the rate of chemical reaction (2), we expand the velocity distribution function $f$ to second order as

$$
f=f^{(0)}+f^{(1)}+f^{(2)}=f^{(0)}\left(1+\phi^{(1)}+\phi^{(2)}\right)
$$

around the local Maxwellian, $f^{(0)}=n(m / 2 \pi \kappa T)^{3 / 2} \exp \left[-m \mathbf{v}^{2} / 2 \kappa T\right]$, with $n$ the density of molecules, $\kappa$ the Boltzmann constant and $T$ the temperature defined from the kinetic energy. Substitution of eq.(4) into eq.(2) leads to

$$
R=R^{(0)}+R^{(1)}+R^{(2)}
$$

up to second order. The zeroth-order term of $R$,

$$
R^{(0)}=\int d \mathbf{v} \int d \mathbf{v}_{1} \int d \boldsymbol{\Omega} \int f^{(0)} f_{1}^{(0)} g \sigma(g)=4 n^{2} \sigma^{2}\left(\frac{\pi \kappa T}{m}\right)^{\frac{1}{2}} e^{-\frac{E^{*}}{\kappa T}},
$$

corresponds to the rate of chemical reaction of the equilibrium theory. Similarly, the firstorder term of $R$ is obtained as

$$
R^{(1)}=\int d \mathbf{v} \int d \mathbf{v}_{1} \int d \boldsymbol{\Omega} \int f^{(0)} f_{1}^{(0)}\left[\phi^{(1)}+\phi_{1}^{(1)}\right] g \sigma(g),
$$

where $R^{(1)}$ does not appear because $\phi^{(1)}$ is an odd functions of $\mathbf{c}$, as will be shown in the next section. The second-order term of $R$, i.e. $R^{(2)}$, is divided into

$$
R^{(2, A)}=\int d \mathbf{v} \int d \mathbf{v}_{1} \int d \boldsymbol{\Omega} \int f^{(0)} f_{1}^{(0)} \phi^{(1)} \phi_{1}^{(1)} g \sigma(g),
$$


and

$$
R^{(2, B)}=\int d \mathbf{v} \int d \mathbf{v}_{1} \int d \boldsymbol{\Omega} \int f^{(0)} f_{1}^{(0)}\left[\phi^{(2)}+\phi_{1}^{(2)}\right] g \sigma(g) .
$$

Since the integrations (8) and (9) have the cutoff from eq.(3), the explicit forms of $\phi^{(1)}$ and $\phi^{(2)}$ of the steady-state Boltzmann equation for hard-sphere molecules are required to calculate $R^{(2, A)}$ and $R^{(2, B)}$, respectively.

\section{EXPLICIT FORM OF THE VELOCITY DISTRIBUTION FUNCTION TO SECOND ORDER}

We introduce the explicit form of the velocity distribution function of the steady-state Boltzmann equation for hard-sphere molecules to second order in the density and the temperature gradient along $x$-axis $[25]$ :

$$
\phi^{(1)}=-\frac{4 J_{x}}{5 b_{11} n \kappa T}\left(\frac{m}{2 \kappa T}\right)^{\frac{1}{2}} \sum_{r \geq 1} r ! b_{1 r} c_{x} \Gamma\left(r+\frac{5}{2}\right) S_{\frac{3}{2}}^{r}\left(\mathbf{c}^{2}\right),
$$

which is an odd function of $\mathbf{c}$, and

$$
\begin{array}{r}
\phi^{(2)}=\frac{4096 m J_{x}^{2}}{5625 b_{11}^{2} n^{2} \kappa^{3} T^{3}}\left\{\sum_{r \geq 2} r ! b_{0 r} \Gamma\left(r+\frac{3}{2}\right) S_{\frac{1}{2}}^{r}\left(\mathbf{c}^{2}\right)+\right. \\
\left.\sum_{r \geq 0} r ! b_{2 r}\left(2 c_{x}^{2}-c_{y}^{2}-c_{z}^{2}\right) \Gamma\left(r+\frac{7}{2}\right) S_{\frac{5}{2}}^{r}\left(\mathbf{c}^{2}\right)\right\},
\end{array}
$$

with the scaled velocity $\mathbf{c} \equiv(m / 2 \kappa T)^{1 / 2} \mathbf{v}$. Here $\Gamma(X)$ represents the Gamma function and $S_{k}^{r}(X)$ is a Sonine polynomial, the definition and the orthogonality properties of which are written in our paper. [25] $J_{x}=-75 b_{11} \kappa^{3 / 2} T^{1 / 2} \partial_{x} T / 64 \pi^{1 / 2} m^{1 / 2} d^{2}$ denotes the steady heat flux with $\partial_{x} \equiv \partial / \partial x$. The numerical values for $b_{1 r}, b_{0 r}$ and $b_{2 r}$ are listed in Table 【. Note that, though we have derived them in forms of fractions, we use them in forms of four significant figures, since the forms of the fractions are too complicated. We have derived each of $\phi^{(1)}$ and $\phi^{(2)}$ to 7 th approximation of Sonine polynomials, though the complete forms of $\phi^{(1)}$ and $\phi^{(2)}$ for hard-sphere molecules are the sums of an infinite series of Sonine polynomials. However, we have confirmed that $\phi^{(2)}$ does not converge to 4 th Sonine approximation but both of $\phi^{(1)}$ and $\phi^{(2)}$ almost converge to 7th Sonine approximation[25], so that, in this letter, we will show the results calculated from $\phi^{(1)}$ and $\phi^{(2)}$ for 7 th approximation of Sonine polynomials. We note that Burnett had determined only $b_{1 r}$ and $b_{2 r}$ to 4 th Sonine approximation: $b_{2 r}$ is related to the second-order pressure tensor. 24] Therefore, we could not calculate the effect 
of steady heat flux on the rate of chemical reaction with the line-of-centers model if we did not derive the explicit forms of $\phi^{(1)}$ and $\phi^{(2)}$, especially $b_{0 r}$ in ref. [25].

In order to compare the results from the steady-state Boltzmann equation with those from the steady-state Bhatnagar-Gross-Krook(BGK) equation and information theory, we also use the explicit forms of $\phi^{(1)}$ and $\phi^{(2)}$ for them. The expressions of $\phi^{(1)}$ and $\phi^{(2)}$ for the steady-state BGK equation can be reduced from the general form of the ChapmanEnskog solution for the steady-state BGK equation to arbitrary order 28, 29], while those for information theory [26] were used by Fort and Cukrowski. 12, 13] We mention that $\phi^{(1)}$ for the steady-state BGK equation is an odd function of $\mathbf{c}$ and identical with that for information theory, while $\phi^{(2)}$ are different from each other.

\section{RESULTS: RATE OF CHEMICAL REACTION}

Inserting $\phi^{(1)}$ and $\phi^{(2)}$ of eqs.(10) and (11) and the corresponding velocity distribution functions for the steady-state BGK equation and information theory into eqs.(8) and (9), and performing the integrations with the chemical reaction cross-section (3), we finally obtain the nonequilibrium effects on the rate of chemical reaction based on the line-of-centers model. The expressions of $R^{(2, A)}$ and $R^{(2, B)}$ become

$$
R^{(2, A)}=\frac{\sigma^{2} m J_{x}^{2}}{\kappa^{3} T^{3}}\left(\frac{\pi \kappa T}{m}\right)^{\frac{1}{2}} e^{-\frac{E^{*}}{\kappa T}}\left\{\sum_{r \geq 0} \alpha_{r}\left(\frac{E^{*}}{\kappa T}\right)^{r}\right\},
$$

and

$$
R^{(2, B)}=\frac{\sigma^{2} m J_{x}^{2}}{\kappa^{3} T^{3}}\left(\frac{\pi \kappa T}{m}\right)^{\frac{1}{2}} e^{-\frac{E^{*}}{\kappa T}}\left\{\sum_{r \geq 0} \beta_{r}\left(\frac{E^{*}}{\kappa T}\right)^{r}\right\},
$$

respectively. The numerical values for $\alpha_{r}$ and $\beta_{r}$ are listed in Tables [I and III respectively. We have found that $R^{(2, B)}$ for the steady-state Boltzmann equation is determined only by the terms of $b_{0 r}$ in $\phi^{(2)}$ of eq.(11) which Burnett 24] had not derived: the terms of $b_{0 r}$ we have derived in ref. [25] are indispensable for the calculation of $R^{(2, B)}$.

The explicit expressions in eqs.(12) and (13) for information theory were already given by Fort and Cukrowski. 12, 13] Though they were interested in forms of $Q_{A} \equiv R^{(2, A)} / R^{(0)}$ and $Q_{B} \equiv R^{(2, B)} / R^{(0)}[12$, 13], in the present letter, we focus on the nonequilibrium effects on the rate of chemical reaction in the forms of $R^{(2, A)}$ and $R^{(2, B)}$. This is because the forms of $Q_{A}$ and $Q_{B}$ for the steady-state Boltzmann equation do not converge even when we adopt 
any higher order approximation of Sonine polynomials. The upper limit of $r$ in eqs.(12) and (13) is directly related to the order of the approximation of Sonine polynomials in eqs.(10) and (11): $S_{k}^{r}\left(\mathbf{c}^{2}\right)$ includes the term of $\mathbf{c}^{2 r}$. We emphasize that, however, the values of $R^{(2, A)}$ and $R^{(2, B)}$ for the steady-state Boltzmann equation converge to 7 th approximation of Sonine polynomials. It should be also mentioned that there appear artificial qualitative differences in both $Q_{A}$ and $Q_{B}$ for the steady-state Boltzmann equation, the steady-state BGK equation and information theory for large $E^{*} / \kappa T$.

The graphical results of $R^{(2)}$ compared with those of $R^{(2, A)}$ are provided in Fig 1 Both of $R^{(2)}$ and $R^{(2, A)}$ in Fig 1 are scaled by $\pi^{1 / 2} d^{2} m^{1 / 2} J_{x}^{2} / \kappa^{5 / 2} T^{5 / 2}$. Note that $R^{(2)}$ is the sum of $R^{(2, A)}$ and $R^{(2, B)}$ in eqs.(12) and (13). As Fig 1) 1 shows, it is clear that $R^{(2, B)}$ plays an essential role for the evaluation of $R^{(2)}$. We have also found that there are no qualitative differences in $R^{(2)}$ of the steady-state Boltzmann equation, the steady-state BGK equation and information theory, while $R^{(2, A)}$ also exhibits a slight deviation from each other. This deviation in $R^{(2, A)}$ would not be observed if we adopted $\phi^{(1)}$ of the steady-state Boltzmann equation for the lowest approximation of Sonine polynomials as in the previous papers [14, 15]. This is because $\phi^{(1)}$ of the steady-state Boltzmann equation for the lowest Sonine approximation is identical with the precise $\phi^{(1)}$ of the steady-state BGK equation and information theory. It should be mentioned that we have found qualitative differences among these theories in some thermodynamic quantities, e.g. the second-order pressure tensor. [25, 30] The nonequilibrium effects on the rate of chemical reaction is an insensitive quantity to the differences among the three theories.

\section{DISCUSSION}

$\phi^{(2)}$ is indispensable for the calculation of the nonequilibrium effects on the rate of chemical reaction, since $R^{(1)}$ does not appear and $R^{(2, B)}$ is remarkably larger than $R^{(2, A)}$ as Fig 1 shows. Thus, the nonequilibrium effect on the rate of chemical reaction will substantiate significance of the second-order coefficients in the solution of the steady-state Boltzmann equation, although their importance has been demonstrated only for descriptions of shock wave profiles and sound propagation phenomena $[31$, 32, 33]. We should emphasize that the calculation of the second-order pressure tensor and the application of it to shock wave profiles and sound propagation do not require the explicit form of the second-order veloc- 
ity distribution function: the second-order coefficients derived by Burnett do not include any terms of $b_{0 r}$ in $\phi^{(2)}$ of eq.(11). 24, 25] The nonequilibrium effects we consider appear mainly in the ranges of small $E^{*} / \kappa T$ where the chemical reaction often occurs, so that our results should be compared with experimental results in the early stage of the chemical reaction 27]; a large additional effect due to some modification of the velocity distribution function because of the chemical reaction itself becomes significant as the chemical reaction proceeds [17, 18].

We also propose a thermometer of a monatomic dilute gas system under steady heat flux. We mean that we can measure the temperature $T$ around a heat bath at $T_{0}$ in the nonequilibrium steady-state system indirectly with the aid of the nonequilibrium effect on the rate of chemical reaction as follows. The nonequilibrium effect on the rate of chemical reaction in the early stage around the heat bath can be measured. Thus, one can compare the experimental result with the theoretical result shown in Fig 1 with $T=T_{0}$. The difference between the former and the latter will indicate that the temperature $T$ around the heat bath is not identical with $T_{0}$, that is, $T=T_{0}+\Delta$. Here $\Delta$ should depend upon the heat flux in general. Substituting this temperature expression into the explicit expressions of $R^{(0)}$ in eq.(6), we obtain a new correction term

$$
R^{\text {(new) }}=\frac{2 n^{2} \sigma^{2} \Delta}{T_{0}}\left(\frac{\pi \kappa T_{0}}{m}\right)^{\frac{1}{2}}\left(1+\frac{2 E^{*}}{\kappa T_{0}}\right) e^{-\frac{E^{*}}{\kappa T_{0}}}
$$

as the nonequilibrium effect on the rate of chemical reaction besides $R^{(2)}$ in Fig 1 with $T=T_{0}$. At last, we can estimate the gap $\Delta$ so as to make the new correction term match the experimental result. For example, if $\Delta$ in eq.(14) is proportional to the heat flux, we will confirm the relevancy of the slip effect 34, 35]. If $\Delta$ in eq.(14) is identical with $2 m J_{x}^{2} / 5 n^{2} \kappa^{3} T_{0}^{2}$, i.e. $1 / T=1 / T_{0}-2 m J_{x}^{2} / 5 n^{2} \kappa^{3} T_{0}^{4}$, the experimental result will agree with the theoretical result from the steady-state Boltzmann equation with the nonequilibrium temperature $\theta=T_{0}$ predicted by Jou et al. [26]. We show the comparison of the theoretical results, i.e. $R^{(2)}$ for the steady-state Boltzmann equation with $T=T_{0}$ and that with $\theta=T_{0}$, in Fig 2, where $R^{(2)}$ is scaled by $\pi^{1 / 2} d^{2} m^{1 / 2} J_{x}^{2} / \kappa^{5 / 2} T_{0}^{5 / 2}$. We note that one can obtain the explicit form of $R^{(2)}$ with $\theta=T_{0}$ expressed as the dashed line in Fig 2 as the sum of $R^{(2)}$ from eqs.(12) and (13) for the steady-state Boltzmann equation with $T=T_{0}$ and $R^{\text {(new) }}$ from eq. (14) with $\Delta=2 m J_{x}^{2} / 5 n^{2} \kappa^{3} T_{0}^{2}$. We have found that there is a significant difference between $R^{(2)}$ with $T=T_{0}$ and that with $\theta=T_{0}$ for small $E^{*} / \kappa T_{0}$. This significant 
difference also appears in $R^{(2)}$ for the steady-state BGK equation and information theory. Similar difference in $R^{(2)}$ for information theory would also follow from the results of Fort and Cukrowski[13], although they were not interested in the ranges of small $E^{*} / \kappa T$. We emphasize that, however, our proposal written in this paragraph differs from that by Fort and Cukrowski[13] which uses a given value measured directly by a thermometer in order to determine $T$ or $\theta$ regardless of the temperature of the heat bath.

\section{acknowledgments}

This study has been supported partially by the Hosokawa Powder Technology Foundation and the Inamori Foundation.

[1] S. Chapman and T. G. Cowling, The Mathematical Theory of Non-uniform Gases, Cambridge University Press, London, 1990.

[2] P. Résibois and M. De Leener, Classical Kinetic Theory of Fluids, A Wiley-Interscience Publication, New York, 1977.

[3] J. L. Lebowitz, E. W. Montroll, The Boltzmann equation, Elsevier Science, New York, 1983.

[4] R. D. Present, Kinetic Theory of Gases, Mcgraw-Hill, New York, 1958.

[5] R. D. Present, J. Chem. Phys. 31 (1959) 747.

[6] B. Schizgal and M. Karplus, J. Chem. Phys. 52 (1970) 4262.

[7] C. W. Pyun and J. Ross, J. Chem. Phys. 40 (1964) 2572.

[8] B. Schizgal, J. Chem. Phys. 55 (1971) 76.

[9] R. Kapral, S. Hudson and J. Ross, J. Chem. Phys. 53 (1970) 4387.

[10] A. S. Cukrowski and J. Popielawski, Acta Phys. Pol. A 70 (1986) 321.

[11] J. Popielawski, J. Chem. Phys. 83 (1985) 790.

[12] J. Fort and A. S. Cukrowski, Chem. Phys. 222 (1997) 59.

[13] J. Fort and A. S. Cukrowski, Acta Phys. Pol. B 29 (1998) 1633.

[14] A. S. Cukrowski and J. Popielawski, Chem. Phys. 109 (1986) 215.

[15] B. C. Eu and K.-W. Li, Physica A 88 (1977) 135.

[16] D. G. Napier and B. D. Shizgal, Phys. Rev. E 52 (1995) 3797. 
[17] B. D. Shizgal and D. G. Napier, Physica A 223 (1996) 50.

[18] A. S. Cukrowski, S. Fritzsche and J. Fort, Chem. Phys. Lett. 341 (2001) 585.

[19] R. D. Present, Proc. Natl. Acad. Sci. USA 41 (1955) 415.

[20] M. A. Eliason and J. O. Hirschfelder, J. Chem. Phys. 30 (1959) 1426.

[21] R. D. Levine and R. B. Bernstein, Molecular Reaction Dynamics and Chemical Reactivity, Oxford University Press, New York, 1987.

[22] R. E. Nettleton, Z. Phys. Chem. 196 (1996) 177.

[23] R. E. Nettleton, J. Phys. Chem. 100 (1996) 11005.

[24] D. Burnett, Proc. Lond. Math. Soc. 40 (1935) 382.

[25] Kim H.-D. and H. Hayakawa, http://arxiv.org/abs/cond-mat/0202003, submitted to J. Phys. Soc. Jpn.

[26] D. Jou, J. Casas-Vazquez and G. Lebon, Extended Irreversible Thermodynamics, Springer, Berlin, 2001.

[27] I. Prigogine and E. Xhrouet, Physica 15 (1949) 913.

[28] A. Santos, J. J. Brey, C. S. Kim and J. W. Dufty, Phys. Rev. A 39 (1989) 320.

[29] C. S. Kim, J. W. Dufty, A. Santos and J. J. Brey, Phys. Rev. A 39 (1989) 328.

[30] Kim H.-D. and H. Hayakawa, http://arxiv.org/abs/cond-mat/0203036, submitted to J. Phys. Soc. Jpn.

[31] J. D. Foch Jr. , Acta Phys. Aust. Suppl. X (1973) 123.

[32] K. A. Fiscko and D. R. Chapman, Rarefied Gas Dynamics Mechanics of Nonequilibrium Processes, edited by E. P. Muntz, D. P. Weaver and D.H. Campbell, American Institute of Aeronautics and Astronautics, Washington, D.C., 1989, p.374.

[33] F. E. Lumpkin III and D. R. Chapman, J. Thermophys. Heat Transfer 6 (1992) 419.

[34] Y. Sone and S. Takata, Trans. Theor. Stat. Phys. 21 (1992) 501.

[35] C. Cercignani, Mathematical Methods in Kinetic Theory, Plenum Press, New York, 1990. 


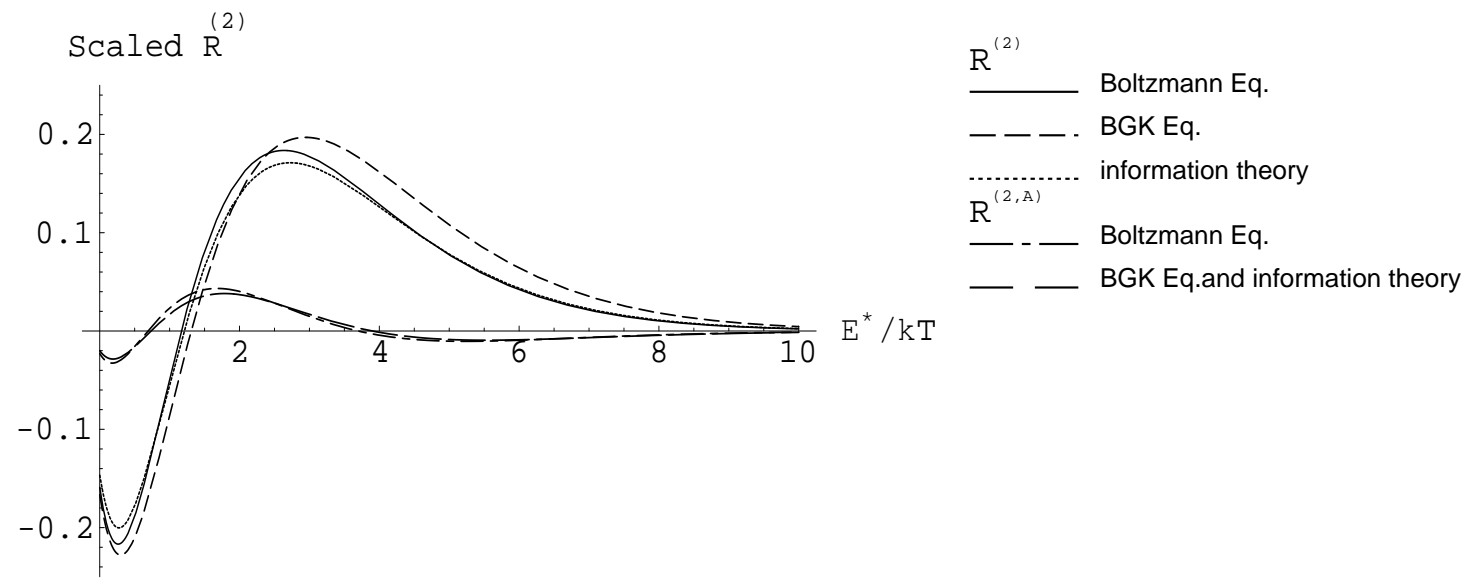

FIG. 1: Scaled $R^{(2)}$ compared to scaled $R^{(2, A)}$ as a function of $E^{*} / \kappa T$ for the line-of-centers model.

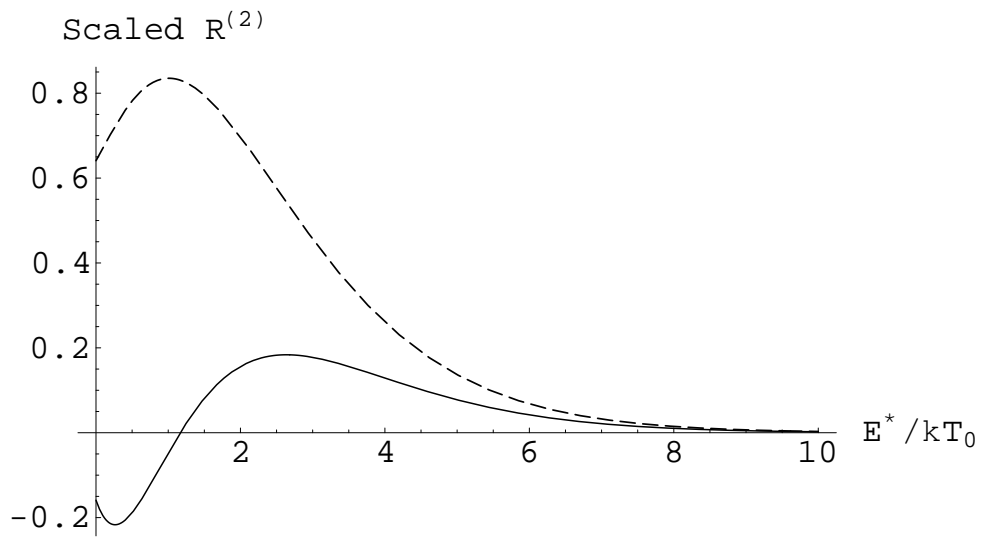

FIG. 2: Comparison of scaled $R^{(2)}$ from the steady-state Boltzmann equation with $T=T_{0}$ (the solid line) and that with $\theta=T_{0}$ (the dashed line) as a function of $E^{*} / \kappa T_{0}$. Note that the former is identical with the result shown by the solid line in Fig.1 with $T=T_{0}$. 
TABLE I: Numerical constants $b_{1 r}, b_{0 r}$ and $b_{2 r}$ in eqs.(10) and (111).

\begin{tabular}{|c|c|c|c|}
\hline \hline$r$ & $b_{1 r}$ & $b_{0 r}$ & $b_{2 r}$ \\
\hline 0 & 0 & 1 & $-3.320 \times 10^{-2}$ \\
\hline 1 & 1.025 & 0 & $-1.276 \times 10^{-1}$ \\
\hline 2 & $4.892 \times 10^{-2}$ & $4.380 \times 10^{-1}$ & $6.414 \times 10^{-2}$ \\
\hline 3 & $3.715 \times 10^{-3}$ & $-5.429 \times 10^{-2}$ & $5.521 \times 10^{-3}$ \\
\hline 4 & $2.922 \times 10^{-4}$ & $-4.098 \times 10^{-3}$ & $4.214 \times 10^{-4}$ \\
\hline 5 & $2.187 \times 10^{-5}$ & $-3.184 \times 10^{-4}$ & $3.106 \times 10^{-5}$ \\
\hline 6 & $1.492 \times 10^{-6}$ & $-2.087 \times 10^{-5}$ & $1.861 \times 10^{-6}$ \\
\hline 7 & $8.322 \times 10^{-8}$ & - & - \\
\hline \hline
\end{tabular}

TABLE II: Numerical constants $\alpha_{r}$ in eq.(12).

\begin{tabular}{|c|c|c|}
\hline$r$ & Boltzmann Eq. & BGK Eq. and Information Theory \\
\hline 0 & $-2.292 \times 10^{-2}$ & $-\frac{1}{50}$ \\
\hline 1 & $-1.448 \times 10^{-1}$ & $-\frac{3}{25}$ \\
\hline 2 & $3.223 \times 10^{-1}$ & $\frac{6}{25}$ \\
\hline 3 & $-9.834 \times 10^{-2}$ & $-\frac{4}{75}$ \\
\hline 4 & $7.919 \times 10^{-3}$ & - \\
\hline 5 & $-6.752 \times 10^{-4}$ & - \\
\hline 6 & $5.298 \times 10^{-5}$ & - \\
\hline 7 & $-3.584 \times 10^{-6}$ & - \\
\hline 8 & $2.039 \times 10^{-7}$ & - \\
\hline 9 & $-9.599 \times 10^{-9}$ & - \\
\hline 10 & $3.613 \times 10^{-10}$ & - \\
\hline 11 & $-1.032 \times 10^{-11}$ & - \\
\hline 12 & $2.110 \times 10^{-13}$ & - \\
\hline 13 & $-2.887 \times 10^{-15}$ & - \\
\hline 14 & $2.352 \times 10^{-17}$ & - \\
\hline 15 & $-8.578 \times 10^{-20}$ & - \\
\hline
\end{tabular}


TABLE III: Numerical constants $\beta_{r}$ in eq.(13).

\begin{tabular}{|c|r|r|r|}
\hline \hline$r$ & Boltzmann Eq. & BGK Eq. & Information Theory \\
\hline 0 & $-1.361 \times 10^{-1}$ & $-\frac{11}{75}$ & $-\frac{19}{150}$ \\
\hline 1 & $-5.094 \times 10^{-1}$ & $-\frac{38}{75}$ & $-\frac{7}{15}$ \\
\hline 2 & $3.968 \times 10^{-1}$ & $\frac{4}{15}$ & $\frac{26}{75}$ \\
\hline 3 & $5.805 \times 10^{-2}$ & $\frac{8}{75}$ & $\frac{4}{75}$ \\
\hline 4 & $-2.811 \times 10^{-3}$ & - & - \\
\hline 5 & $1.039 \times 10^{-4}$ & - & - \\
\hline 6 & $-1.808 \times 10^{-6}$ & - & - \\
\hline \hline
\end{tabular}

